\title{
Heart failure in COVID-19 pandemic: Challenging management issues
}

\author{
Soumya Patnaik ${ }^{1}$, and Amar Narayan Patnaik ${ }^{2, *}$ \\ ${ }^{1}$ Fellow, Division of Cardiovascular Disease, University of Texas Health Sciences Center, Houston; Texas, USA \\ ${ }^{2}$ Senior Consultant Cardiologist, Star Hospitals, Hyderabad-500029, India
}

\begin{abstract}
Over these 12 months of coronavirus disease 2019 (COVID-19) pandemic, the patients of heart failure (HF) showed higher risk of severe disease and increased mortality. Their evaluation and treatment when additionally affected with COVID-19 posed many new challenges, as one condition can potentiate the other and there is considerable overlap of their presenting features. The pandemic also impacted the existing health care systems with changed priorities. Known HF patients becoming worse after acquiring the COVID-19 infection or the new development of acute cardiac complications in persons without any prior heart disease- both are novel therapeutic issues which have no perfect answers for want of adequate data and randomised trials. Newer suggestions are emerging in the management of HF with concomitant COVID-19 from experience from various sources. We have to keep our minds open and learn every day.
\end{abstract}

Keywords: heart failure; coronavirus; COVID-19; management; SARS-CoV-2

*Corresponding author: Dr. AN. Patnaik, MD., Senior Consultant Cardiologist, Star Hospitals, Road No.10, Banjara Hills, Hyderabad500034. Tel.: 9490421969; Email: anpatnaik@starhospitals.in

Received 5 October 2020; Revised 4 November 2020; Accepted 11 November 2020; Published 19 November 2020

Citation: Patnaik S, Patnaik AN. Heart failure in COVID-19 pandemic: Challenging management issues. J Med Sci Res. 2020; 8(S1):74-80. DOI: http://dx.doi.org/10.17727/JMSR.2020/8S1-9

Copyright: (C) 2020 Patnaik S et al. Published by KIMS Foundation and Research Center. This is an open-access article distributed under the terms of the Creative Commons Attribution License, which permits unrestricted use, distribution, and reproduction in any medium, provided the original author and source are credited.

\section{Introduction}

Coronavirus disease 2019 (COVID-19) pandemic is about to complete one-year of its emergence into our lives. First of all, it jeopardised the existing health care system including the cardiac services with changed priorities all over the globe. It is now clear that this viral disease affects multiple systems with frequent cardiac manifestations with enhanced degree of morbidity and mortality. The treatment of ischemic syndromes, decisions on revascularisation procedures and cardiac surgeries, management of patients with heart failure including the heart transplant recipients and many other cardiac care issues became more challenging in these pandemic months.

Zhou et al found that $23 \%$ of their 191 patients of COVID-19 inpatients had heart failure, which was as 
high as 52\% among the non-survivors [1]. Inciardi and Chen also reported similar trends of heart failure incidence among the Covid-19 patients [2, 3]. Currently there are no randomised trials to elucidate rationale therapies and HF management algorithms, due to the acuteness of the pandemic and lack of prior experience with COVID-19 infections. Lack of clarity or uniformity prompted us to review available literature on the impact of COVID-19 on heart failure management.

\section{Possible mechanisms of cardiac dysfunction and heart failure}

Heart Failure is common enough to be encountered at different stages in the course of the COVID-19 pandemic. There are multiple ways by which a prior HF patient may worsen, or a new onset HF can occur in a healthy individual.

\section{Acute cardiac injury}

Elevated cardiac troponins or new ECG or echocardiographic abnormalities are considered to indicate acute cardiac injury. Wang D et al showed that as many as $7.2 \%$ of all COVID-19 patients $(22 \%$ of those requiring ICU admission) presented with acute cardiac injury [4]. However, National Health Commission of China reported that as many as $12 \%$ of patients, without prior CV disease or any cardiac arrest can show evidence of acute cardiac injury [5]. A level of $>99$ percentile URL hs-cTnl was noted in $46 \%$ of non-survivors versus $1 \%$ among the survivors in Wuhan study [1]. Those with higher levels of troponins had more complications and higher mortality. The need for mechanical ventilation and circulatory support was higher in them [6-8]. Studies varied in their definition of cardiac injury and timing of the sampling, based on cTn levels; it is suggested to have serial measurements and report their data in a universally accepted template designed by Sandoval et al [9].

\section{Generalised inflammation and acute myocarditis}

In advanced stages, there can be a state of cytokine storm with increased IL-3, IL-6 and IL-7, granulocytecolony stimulating factor, interferon-gamma inducible protein-10, monocyte chemoattractant protein 1, macrophage inflammatory protein 1-alfa and tumour necrosis factor-alfa and ferritin. The inflammatory status and production of cytokines secondary to infection increases blood viscosity and coagulopathy. Endothelial dysfunction, fluid and electrolyte imbalances add up resulting in decompensation of the sub-clinical heart failure $[10$, 11].

Acute myocarditis can manifest as a part of generalised inflammatory state or independent of it. In sporadic cases, histopathological confirmation showed low-grade inflammation with non-specific myocardial changes and low or absent myocyte necrosis [12-14]. Among those with elevated TnT or ECG abnormalities suggestive of acute cardiac injury, a small percentage of patients presented with florid symptoms of myocarditis with markedly raised inflammatory markers or MRI evidence for inflammation, most often, with a rapidly progressive down-hill course. Tomasoni et al and Luetkens et al observed generalised myocardial oedema in absence of LGE at cardiac MRI and these patients had normal echocardiogram findings and elevated biomarkers of cardiac injury $[15,16]$.

While some of those presenting with myocarditis were a component of the cytokine storm with multiorgan dysfunction, a few were isolated myocarditis or presented with associated respiratory failure. Most of such cases succumbed fatally after a period of circulatory shock and arrhythmias $[17,18]$.

\section{Endothelitis}

In these last 12 months it was observed that there is sharp rise in cases of STEMI and when taken up for primary PCI, the operators were surprised to see no significant culprit lesion in most cases. Some of these could be explained as a type of endothelitis involving the microcirculation in COVID-19 infection. This could be an early stage of systemic inflammatory response to infection especially in those with preexisting cardiovascular disease (CVD) [19].

\section{Hypoxemia induced myocardial dysfunction}

Pneumonia is a predominant manifestation of COVID-19 affection. Extensive pneumonia leads to hypoxemia and subsequent myocardial damage. There can be pulmonary hypertension and rightheart dysfunction as well. Use of elevated positive endexpiratory pressure during mechanical ventilation also adds to the right ventricular afterload and wallstress. 


\section{Stress-cardiomyopathy}

Jabri et al studied the incidence of stresscardiomyopathy (SCMP) among 1914 ACS patients with COVID test negative reports during the COVID pandemic compared to historical controls from 2018 and 2019. The incidence of SCMP was 7.8\% (vs 1.5\%). The length of hospital-stay was longer but there was no difference in mortality or 30-day re-hospitalisation rates. Minhas documented a case of stress-cardiomyopathy in the setting of COVID-19, which he claims as the first ever such case in USA [29, 30]. These observations suggest that in a few cases, severe stress itself can produce cardiomyopathy with or without getting actual COVID-19 infection $[20,21]$.

\section{Worsening of prior HF}

Worsening heart failure in previously diagnosed patients is not an uncommon event, most requiring ICU admission. HF was observed in about 20$30 \%$ of the COVID-19 patients admitted to intensive care units, as reported in various centres [1-3]. Myocarditis, acute cardiac injury, cardiac arrhythmias, thrombotic events, fluid imbalances, plaque instability or disruption in guidelines directed medical therapies can all precipitate a decompensated state in a previously stabilised $\mathrm{HF}$ patient.

\section{Type of presentations}

It is better to consider presentation and prognosis of heart failure in three clinical scenarios-

(a) In those without any known prior cardiac affection,

(b) Those with prior stable heart failure who decompensated after COVID-19 infection,

(c) Those with HF preserved EF.

\section{(a) No prior heart disease}

Cardiac affection is not unique to the current COVID19 pandemic. During the severe acute respiratory syndrome (SARS), Middle East respiratory syndrome (MERS) and Influenza epidemics in the past, acute cardiac injury was noted in a good number of patients resulting in the development of heart failure, arrhythmias, and sudden cardiac death [22]. Severe COVID-19 infection can affect the cardiovascular system even in those without prior heart disease and can manifest as acute cardiac injury, myocarditis, acute heart failure, myocardial infarction (Type I/
II), acute pulmonary embolism, cardiac arrhythmias and stress-induced cardiomyopathy [1].

\section{(b) Prior HF present}

Viral infections like Influenza were known to precipitate acute decompensation in heart failure patients [23]. In a large study from Spain, among 3080 positive RT-PCR patients 153 (4.9\%) had CHF. Of these $98(64.5 \%)$ had some degree of LV systolic dysfunction prior to diagnosis. The rest 54 had preserved or normalised LVEF (following treatment) or had valvular heart disease with no systolic dysfunction. The former had more CV risk factors and co-morbidities and were of advanced age. They showed higher troponins and pro-BNP, higher incidence of thrombotic events and higher mortality. The authors observed that withdrawal of GDMT was associated with precipitation of acute decompensation and higher mortality in those who had prior HF [24]. In a study of 6,439 patients those who had prior HF, had longer hospital stay, increased risk of mechanical ventilation and mortality compared those who never reported HF prior to their admission regardless of EF at admission [25].

Chatrath et al in a retrospective analysis of 134 hospitalised patients of chronic heart failure of which 40 tested positive for COVD-19 infection. Top co-morbidities were comparable between positive and negative groups. Among the patients who tested positive the mortality was almost 5 times greater at $50.0 \%$ vs $10.6 \%$ (RR 4.70 ). COVID-19 pneumonia and decompensated heart failure was the cause of death in $40 \%$ of them. Evidence for myocardial injury and need for Oxygen and treatment for superadded infection was higher in the positive group. The authors attributed the excess morbidity and mortality to excess of inflammatory and immunological pathways, that lead to cardiac dysfunction and renal hypoperfusion [26]. COVID-19 infection manifests in severe form in the elderly HF patients with increased mortality dure to reduced immunity and general frailty and age-related disabilities.

\section{(c) Heart failure with preserved EF}

This type of presentation in patients with COVID19 disease is possible by direct viral infiltration, inflammation, or cardiac fibrosis or by unmasking of pre-existing HFpEF. Direct myocardial injury is evidenced by elevated biomarkers, cardiac 
imaging, and autopsy reports. The release of proinflammatory cytokines like IL- 1 and IL- 6 favour the role of inflammation. At least 2 echocardiographic studies have shown that in nearly $90 \%$ of hospitalised patients the LVEF was normal but there could be RV dysfunction and LV diastolic dysfunction. This may be due to hypoxemic respiratory failure and thrombotic events [27]. Higher morbidity in the diabetic and elderly patients suggests possible metabolic injuries in the development of HFpEF [28].

\section{Management of heart failure}

Pneumonia and pulmonary oedema can be confused with each other and also may co-exist in the same patient. Early admission of all suspected cases to ICU has to be decided by a designated COVID physician without wastage of precious time as patients can deteriorate very rapidly. Evaluation includes extensive and repeated imaging studies and tests for the presence of the virus. Besides basic cardiac, pulmonary, renal, and hepatic evaluation tests like D-dimer, platelet count, serum ferritin, CRP, troponins, BNP etc are warranted. Evaluation of the thrombotic potential and the pro-inflammatory markers is of paramount importance in heart failure patients affected with COVID-19. Lymphopenia, acute kidney injury and elevated LDH, liver enzymes, CRP and serum ferritin, raised PT, troponins; creatinine kinase and D-dimer are predictors of cardiac affection and bad prognosis [1].

Like pre-COVID days the primary focus in acute decompensated heart failure is the enhancement of hemodynamic status through reduction of vascular congestion, improving the preload, after-load, and the ventricular contractility. Correction of hypoxemia, anaemia, infection, electrolytes disturbances and the fluid management are principal concerns. Judicious diuretic, inotropic and vasodilator use, CPAP/ BIPAP/timely intubation enhances recovery from the decompensated state, irrespective of the COVID19 status.

\section{Pharmacotherapy of HF}

Diuretics, RAS inhibitors, beta-blockers and MRAs continue to be the main drugs in the conventional treatment of HF even in COVID-19 patients.

(1) Diuretics: Critical care physicians and nephrologists share the responsibility for proper fluid and electrolyte balance in each patient. Their inputs have to be respected while giving diuretics for $\mathrm{HF}$ management. Hypotension has to be investigated for any precipitating factor like low fluid intake, fever, use of NSAIDS, GI bleed or septic shock or any drug -effect. Invasive monitoring has to be decided on case to case basis by the team.

(2) RAS inhibitors: Those who are on ACEI/ ARBs/ARNI have to continue the previous medications unless hypotension or renal derangements warrant dose reduction or temporary stoppage. Based on a single study, initially it was debated whether ACEI/ARBs make the host cells susceptible to COVID-19 infection through an up-regulation of ACE2 [29]. Subsequent observations and analysis confirmed that there is no increase in adverse effects in patients on continued ACEI/ARBs and in fact such RAS inhibition was protective to lung inflammation at a later stage of COVID19 infection [30].

Almost every professional body advised the continuation of ACEI/ARBs as before for hypertension and heart failure. Hypotension or worsening renal function should be the main indication for stoppage or reduction of dose of these cornerstone molecules [31-33].

(3) Beta-blockers: Metoprolol, bisoprolol, carvedilol, or nebivolol are the approved betablockers for heart failure; but they should be used with caution in presence of hemodynamic instability. Drugs like lopinavir/ritonavir or darunavir are also known to decrease heart rates and may cause hypotension and they have to be used judiciously. It is suggested that carvedilol could have unique anti-cytokine properties; but it not been recommended to prefer it over the other three [34].

\section{Management of other systems and co- morbidities}

Management of critically ill patient of HF in the setting of COVID-19 infection in the high definition units is truly a multidisciplinary teamwork calling the services of physicians of other specialities, the critical care physician being the co-ordinator. Pulmonary disease is the predominant affection in many COVID-19 patients. The pulmonologist, ICU 
specialist and anaesthesiologist play crucial role in decision making. Hypoxic respiratory failure and hypoxic cardiac injury can coexist. HF with pulmonary oedema can predispose or worsen the pulmonary complications and lead to enhancement of intensity of mechanical respiratory support. The aim is to achieve Sp02 >95\% (In COPD >90\%). Initially standard oxygen therapy, venturi mask or mask with reservoir bag is used. When high flow oxygen does not help, non-invasive ventilation and prone positioning may be considered. Next escalation step is endo-tracheal intubation and invasive mechanical ventilation with lung protective strategies like low tidal volume (6-8 $\mathrm{ml} / \mathrm{kg}$ ideal body weight) and lowlevel airway platform pressures $<30 \mathrm{~cm} \mathrm{H} 2 \mathrm{O}[10,35]$. Acute kidney injury can be seen in about one-third of the ICU admitted patients. Most of them may need renal replacement therapy of which ultra-filtration may be best for diuretic resistant HF patients [36]. Hemofiltration can effectively control inflammatory response, reduce the offending factors in the blood and minimise the tissue injury in this critical stage of cytokine storm in COVID-19 affection [37].

Anti-viral drugs, immune-modulatory agents and steroids have to be used based on the current evidence and local institutional protocols as there are some controversies and newer updates are constantly pouring in. It is important to remember that some antiviral agents can cause myocardial dysfunction, QTc prolongation and cardiotoxicity as well.

\section{Use of MCS and ECMO in cardiogenic shock}

After oxygenation is taken care, intra-aortic balloon pump can be the initial choice for patients in borderline shock states. If they do not provide adequate support, micro-axial systems like Impella may be tried which adequately unload the failing ventricle and reduce cardiac inflammation. Next in the upgradation is veno-venous extracorporeal membrane oxygenation (ECMO) which is both expensive and not available in most centres. In presence of decreased $\mathrm{CO}$ and depressed LV contractility one may prefer the Veno-arterial ECMO and additional respiratory failure warrants a hybrid ECMO. Zheng et al cautioned about the potential for reduction of some classes of lymphocytes and inflammatory activation in patients on ECMO in COVID-19 patients [38-41].

\section{Advanced therapies \\ Heart transplant program}

ACC published the poll results of impact of the COVID-19 pandemic on advanced heart failure and heart transplantation, which was conducted in August, 2020 involving 47 adult program-physicians from 12 countries. Nearly $80 \%$ was altered their approach to the program priorities. One in five stopped the program at some time or became more selective. There was overall decrease in heart transplant volumes. COVID-19 related mortality in heart transplant recipients was $25 \%[42,43]$.

Managing the heart transplant recipients can be quite challenging. In heart transplant recipients, immunosuppressive drugs can cause lymphopenia which may be further enhanced by COVID19 infection. ISHLT guidelines suggested their temporary with-holding in in sever presentations of COVID-19 infection. The anti-viral agents can interfere with action of cyclosporine. It is also possible to misdiagnose COVID-myocarditis to be cardiac transplant rejection. Gene-profiling or donor-derived cell free DNA test can differentiate the two entities [44].

\section{Left ventricular assist devices}

Patients on left ventricular assist devices (LVADs) are prone for various infections and they have to be guarded against COVID-19 as well. The device may need tele-remote programming. In those on long term LVADs can have elevated cytokines even without COVID-19 affection. This has a bearing in evaluation of a COVID-19 positive patient. Increased potential for thrombosis and appropriate anticoagulation may pose additional challenges in these patients. These patients if contract COVID-19 disease can be at higher risk for right heart failure due to hypoxia and probable high output syndrome [44].

\section{Development of updated guidelines}

The recent publication of position paper by Chinese Heart Failure association and the European Society of Cardiology is praise -worthy exercise to put most of the existing data in the right prospective and formulate some suggestions for the management of current issues in management of HF in the COVID19 pandemic. Emergence of guidelines has to wait until more experience is shared through standard publications involving larger number of patients [44]. 
For chronic management of discharged patients, tele-consultations and physician-guided patient self-management programs have been implemented at some centres to reduce hospital visits and admissions. Implantable sensors for remote PA pressure monitoring can be of particular benefit in these unprecedented times.

\section{Conclusions}

The 12-month old COVID-19 pandemic posed unprecedented challenges to the practice of public health and clinical medicine; HF management is no exception. Known HF patients becoming worse after acquiring the COVID-19 infection or the new development of acute cardiac complications in persons without any prior heart disease- both are novel therapeutic issues which have no perfect answers for want of adequate data and randomised trials. Many current practices and expert recommendations in the management of HF with concomitant COVID-19 are bound to be modified in the days ahead with further data and experience. We have to update ourselves every day.

\section{Conflicts of interest}

Authors have no conflict of interest.

\section{References}

[1] Zhou F, Yu T, Du R, Fan G, Liu Y, et al. Clinical course and risk factors for mortality of adult inpatients with COVID-19 in Wuhan, China: a retrospective cohort study. Lancet. 2020; 395(10229):1054-1062.

[2] Inciardi RM, Adamo M, Lupi L, Cani DS, Di Pasquale M, et al. Characteristics and outcomes of patients hospitalized for COVID-19 and cardiac disease in northern Italy. Eur Heart J. 2020; 41(19):1821-1829.

[3] Chen T, Wu D, Chen H, Yan W, Yang D, et al. Clinical characteristics of 113 deceased patients with coronavirus disease 2019: retrospective study. BMJ. 2020; 368:m1091.

[4] Wang D, Hu B, Hu C, Zhu F, Liu X, et al. Clinical characteristics of 138 hospitalized patients with 2019 novel coronavirusinfected pneumonia in Wuhan, China. JAMA. 2020; 323(11):1061-1069.

[5] Zheng Y, Ma Y, Zhang J, Xie X. COVID-19 and the cardiovascular system. Nat Rev Cardiol. 2020; 17(5):259-260.

[6] Shi S, Qin M, Shen B, Cai Y, Liu T, et al. Association of cardiac injury with mortality in hospitalized patients with COVID19 in Wuhan, China. JAMA Cardiol. 2020; 5(7):802-810.

[7] Guo T, Fan Y, Chen M, Wu X, Zhang L, et al. Cardiovascular implications of fatal outcomes of patients with coronavirus disease 2019 (COVID-19). JAMA Cardiol. 2020; 5(7):811818.

[8] Shi S, Qin M, Cai Y, Liu T, Shen B, et al. Characteristics and clinical significance of myocardial injury in patients with severe coronavirus disease 2019. Eur Heart J. 2020; 41(22):2070-2079.
[9] Sandoval Y, Januzzi J, Jaffe AS. Cardiac troponin for the diagnosis and risk-stratification of myocardial injury in COVID-19: JACC review topic of the week. J Am Coll Card. 2020; 76(10):1244-1258.

[10] Mehra MR, Ruschitzka F. COVID-19 illness and heart failure: a missing link? JACC Heart Fail. 2020; 8(6):512-514.

[11] Mehta P, Mcauley DF, Brown M, Sanchez E, Tattersall RS, et al. Correspondence COVID-19: consider cytokine storm syndromes and immunosupression. Lancet. 2020; 395(10229):1033-1034.

[12] Tavazzi G, Pellegrini C, Maurelli M, Belliato M, Sciutti F, et al. Myocardial localization of coronavirus in COVID-19 cardiogenic shock. Eur J Heart Fail. 2020; 22(5):911-915.

[13] Kim IC, Kim JY, Kim HA, Han S. COVID-19-related myocarditis in a 21-year-old female patient. Eur Heart J. 2020; 41(19):1859.

[14] Zhou R. Does SARS-CoV-2 cause viral myocarditis in COVID19 patients? Eur Heart J. 2020; 41(22):2123.

[15] Tomasoni D, Italia L, Adamo M, Inciardi RM, Lombardi CM, et al. COVID-19 and heartfailure: from infection to inflammation and angiotensin II stimulation. Searching for evidence from a new disease. Eur J Heart Fail. 2020; 22(6):957-966.

[16] Luetkens JA, Isaak A, Zimmer S, Nattermann J, Sprinkart AM, et al. Diffuse myocardial inflammation in COVID-19 associated myocarditis detected by multiparametric cardiac magnetic resonance imaging. Circ Cardiovasc Imaging. 2020; 13(5):e010897.

[17] Chen C, Zhou Y, Wang DW. SARS-CoV-2: a potential novel etiology of fulminant myocarditis. Herz. 2020; 45(3):230232.

[18] Ruan Q Yang K, Wang W, Jiang L, Song J. Clinical predictors of mortality due to COVID-19 based on an analysis of data of 150 patients from Wuhan, China. Intensive Care Med. 2020; 46(5):846-848.

[19] Varga Z, Flammer AJ, Steiger P, Haberecker M, Andermatt R, et al. Endothelial cell infection and endothelitis in COVID-19. Lancet. 2020; 395(10234):1417-1418.

28.Hu H, Ma F, Wei X, Fang Y. Corona-virus fulminant myocarditis saved with glucocorticoid and human immunoglobulin. Eur Heart J. 2020; ehaa190.

[20] Jabri A, Kalra A, Kumar A, Alameh A, Adroja S, et al. Incidence of stress cardiomyopathy during the Coronavirus disease 2019 Pandemic. JAMA Netw Open. 2020; 3(7):e2014780.

[21] Minhas AS, Scheel P, Garibaldi B, Liu G, Horton M, et al. Takotsubu syndrome in the setting of COVID-19. JACC Case Rep. 2020; 2(9):1321-1325.

[22] Li SS, Cheng CW, Fu CL, Chan YH, Lee MP, et al. Left ventricular performance in patients with severe acute respiratory syndrome: a 30-day echocardiographic follow-up study. Circulation. 2003; 108(15):1798-1803.

[23] Panhwar MS, Kalra A, Gupta T, Kolte D, Khera S, et al. Effect of influenza on outcomes in patients with heart failure. JACC Heart Fail. 2019; 7(2):112-117.

[24] Rey JR, Caro-Codon J, Rosillo S, Iniesta AM, CastrejónCastrejón S, et al. Heart Failure in COVID-19 patients: prevalence, incidence, and prognostic implications. Eur J Heart Fail. 2020; 24:10.1002/ejhf.1990.

[25] Alvarez-Garcia J, Lee S, Gupta A, Joshi AA, Rivas-Lasarte M, et al. Prognostic Impact of Prior Heart Failure in Patients Hospitalized With COVID-19. J Am Coll Card. 2020; 76(20):2334-2348. 
[26] Chatrath N, Kaza N, Pabari PA, Fox K, Mayet J, et al. The effect of concomitant COVID-19 infection on outcomes in patients hospitalized with heart failure. ESC Heart Fail. 2020; 10.1002/ehf2.13059.

[27] Szekely Y, Lichter Y, Taieb P, Banai A, Hochstadt A, et al. The spectrum of cardiac manifestations in coronavirus disease 2019 (COVID-19) - a systematic echocardiographic study. Circulation. 2020; 142(4):342-353.

[28] Freaney PM, Shah SJ, Khan SS. COVID-19 and Heart failure with preserved ejection fraction. JAMA. 2020; 324:14991500.

[29] Ferrario CM, Jessup J, Chappell MC, Averill DB, Brosnihan $\mathrm{KB}$, et al. Effect of angiotensin-converting enzyme inhibition and angiotensin II receptor blockers on cardiac angiotensinconverting enzyme 2. Circulation. 2005; 111(20):26052610.

[30] Losartan for patients with COVID-19 not requiring hospitalization-full text view-ClinicalTrials.gov: Accessed on 28 Mar 2020 from: https://clinicaltrials.gov/ct2/show/ NCT04311177

[31] Yang X, Yu Y, Xu J, Shu H, Xia J, et al. Clinical course and outcomes of critically ill patients with SARS-CoV-2 pneumonia in Wuhan, China: a single-centred, retrospective, observational study. Lancet Respir Med. 2020; 8(5):475481.

[32] Peng YD, Meng K, Guan HQ Leng L, Zhu RR, et al. Clinical characteristics, and outcomes of 112 cardiovascular disease patients infected by 2019-nCoV. Zhonghua Xin Xue Guan Bing Za Zhi. 2020; 48(6):450-455.

[33] Mancia G, Rea F, Ludergnani M, Apolone G, Corrao G. Reninangiotensin-aldosterone system blockers and the risk of Covid-19. N Engl J Med. 2020; 382(25):2431-2440.

[34] Pauschinger M, Rutschow S, Chandrasekharan K, Westermann D, Weitz A, et al. Carvedilol improves left ventricular function in murine coxsackievirus-induced acute myocarditis association with reduced myocardial interleukin-1beta and MMP-8 expression and a modulated immune response. Eur J Heart Fail. 2005; 7(4):444-452.

[35] Poston JT, Patel BK, Davis AM. Management of Critically Ill Adults with COVID-19. JAMA. 2020; 323(18):1839-1841.

[36] Richardson S, Hirsch JS, Narasimhan M, Crawford JM, McGinn $\mathrm{T}$, et al. Presenting characteristics, comorbidities, and outcomes among 5700 patients hospitalized with COVID19 in the New York City Area. JAMA. 2020; 323(20):20522059.

[37] Tschope C, Linthout SV, Klein O, Mairinger T, Krackhardt F, et al. Mechanical unloading by fulminant myocarditis: LVIMPELLA, ECMELLA, BI-PELLA, and PROPELLA concepts. J Cardiovasc Transl Res. 2019; 12(2):116-123.

[38] Ramanathan K, Antognini D, Combes A, Paden M, Zakhary $\mathrm{B}$, et al. Planning, and provision of ECMO services for severe ARDS during the COVID-19 pandemic and other outbreaks of emerging infectious diseases. Lancet Respir Med. 2020; 8(5):518-526.

[39] Crespo-Leiro MG, Metra M, Lund LH, Milicic D, Costanzo MR, et al. Advanced heart failure: a position statement of the Heart Failure Association of the European Society of Cardiology. Eur J Heart Fail. 2018; 20(11):1505-1535.

[40] Zheng M, Gao Y, Wang G, Song G, Liu S, et al. Functional exhaustion of antiviral lymphocytes in COVID-19 patients. Cell Mol Immunol. 2020; 17(5):533-535.

[41] Henry BM. COVID-19, ECMO, and lymphopenia: a word of caution. Lancet Respir Med. 2020; 8(4):e24.
[42] DeFilippis EM, Reza N, Donald E, Givertz MM, Lindenfeld J, et al. Considerations for heart failure care during the COVID-19 pandemic. JACC Heart Fail. 2020; 8(8):681-691.

[43] Latif F, Farr MA, Clerkin KJ, Habal MV, Takeda K, et al. Characteristics and outcomes of recipients of heart transplant with coronavirus disease 2019. JAMA Cardiol. 2020; e202159.

[44] Zhang Y, Stewart Coats AJ, Zheng Z, Adamo M, Ambrosio G, et al. Management of heart failure patients with COVID-19: A joint position paper of the Chinese Heart Failure Association \& National Heart Failure Committee and the Heart Failure Association of the European Society of Cardiology. Eur J Heart Fail. 2020; 22(6):941-956. 